\title{
REPERTORIO INGRESA AL ÍNDICE BIBLIOGRÁFICO NACIONAL
}

Dario Cadena Rey $M D^{*}$

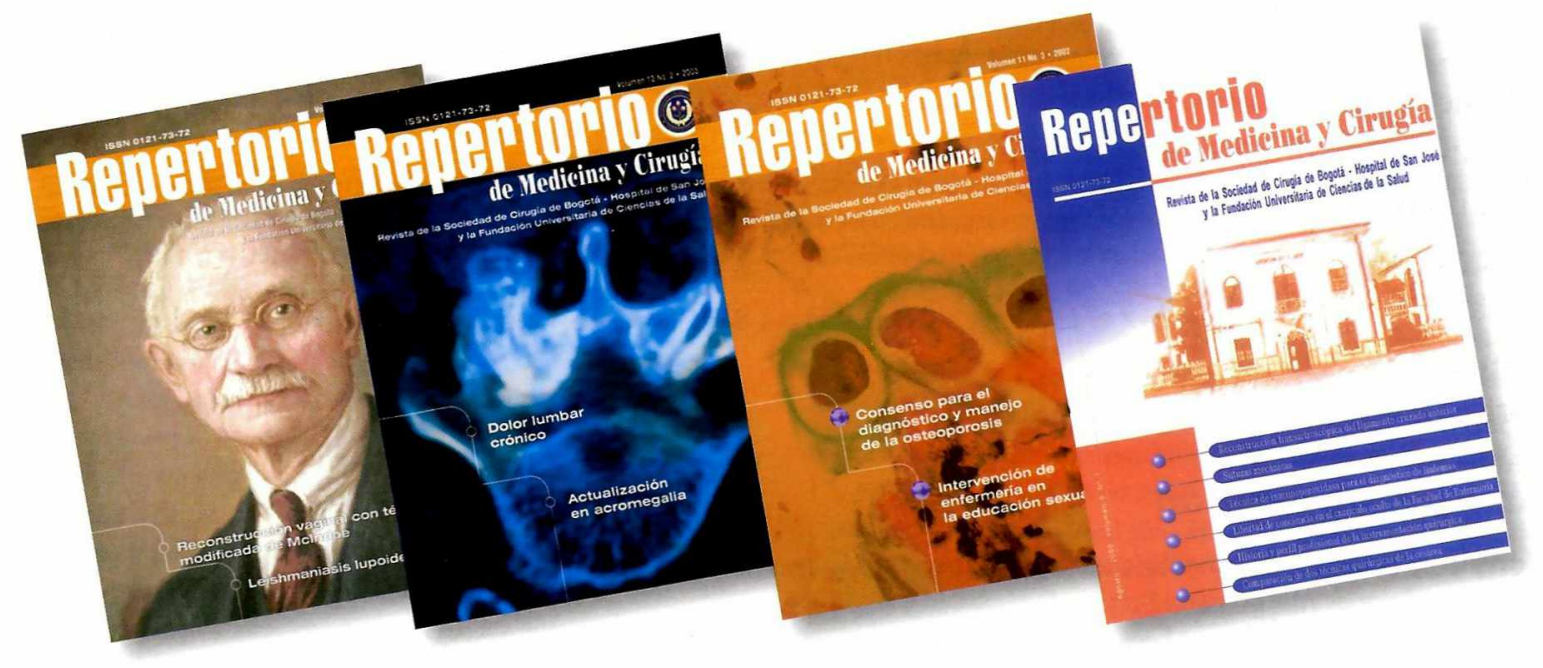

s muy grato comunicarles a nuestros colaboradores y lectores la inclusión del Repertorio de Medicina y Cirugía al selecto grupo de revistas especializadas nacionales indexadas. El Instituto Colombiano para el Desarrollo de la Ciencia y la Tecnología, COLCIENCIAS, adelantó el proceso de normalización y verificación de los datos que integramos a la Base Bibliográfica Nacional, cuyo resultado confirma el cumplimiento de las condiciones pertinentes y por tanto ha sido incluida en el Índice Bibliográfico Nacional, Publindex I - Actualización 2006, en la categoría C con vigencia hasta diciembre de 2007.

Para las revistas científicas es de vital importancia pertenecer al Sistema Nacional de Indexación, pues es el reconocimiento de que cumplen con los criterios de calidad al recolectar y difundir los resultados de las investigaciones nacionales importantes. Esta información especializada le sirve a la comunidad científica para profundizar sus conocimientos sobre las distintas patologías de nuestra región.

Las revistas científicas admitidas al Índice Bibliográfico deben tener determinadas características y cumplir las condiciones de clasificación. Además de las normas generales que rigen para toda revista, hay ciertas especificaciones que diferencian a aquellas de carácter científico, como son el comité editorial y su editor, responsables de la calidad del contenido, quienes someten todos los artículos a la evaluación por pares, cuyos juicios son determinantes para su publicación. Así, las investigaciones quedan abiertas a la opinión de las comunidades de especialistas nacionales y extranjeras, quienes estudian y valoran la calidad de los artículos de la revista.

Dentro de los requisitos básicos para que una revista sea clasificable están entre otros responsabilizarse de la veracidad de la información, tener una existencia certificada según las normas internacionales ISSN (Internacional Standard Serial Number), asegurar una periodicidad declarada y respetada, certificar los niveles de circulación y distribución, así como proveer colecciones completas a la Biblioteca Nacional, la Biblioteca Central de la Universidad Nacional de Colombia y la Biblioteca del Congreso. La estabilidad de la publicación está avalada por su antigüedad y el estricto cumplimiento de las fechas de publicación. La óptima calidad editorial no solo está dada por su contenido, sino en la impresión y los requisitos técnicos. 
E1 Índice Bibliográfico Nacional Publindex considera que los documentos publicados deben corresponder a la siguiente tipología: 1) artículo de investigación científica y tecnológica, que presenta los resultados originales de proyectos de investigación con introducción, metodología, resultados y conclusiones; 2) artículo de reflexión, investigación desde el punto de vista crítico, analítico o interpretativo sobre un tema específico, recurriendo a fuentes originales; 3) artículo de revisión, investigación sobre los avances y tendencias de la ciencia y la tecnología con revisión bibliográfica de por lo menos 50 referencias; 4) artículo corto, información preliminar o parcial de una investigación que requiere pronta difusión; 5) reporte de caso, información de las experiencias en un caso específico, con documentación bibliográfica de casos análogos, y 6 ) revisión de tema, resultado del análisis crítico de la literatura sobre un tema en particular. En Repertorio tenemos una interesante sección sobre historia de la medicina, a veces con recuento histórico de un proceso y en otras ocasiones el análisis crítico de un artículo científico publicado hace más de 50 años.

El primer número de Repertorio de Medicina y Cirugía salió a la luz pública el 15 de octubre de 1909 , bajo la dirección del profesor José María Montoya. En su editorial convocó al cuerpo médico del hospital, de Bogotá y de los Departamentos para que sus observaciones y trabajo diario contra el dolor y la enfermedad, se dieran a conocer en las columnas de la nueva publicación.

Después de sortear numerosas vicisitudes se imprime el último número en 1952, cuando el Hospital de San José se convierte en la sede de la recién fundada facultad de medicina de la Universidad Javeriana, que dura hasta 1964, cuando se traslada al Hospital de San Ignacio. En 1965 la Sociedad de Cirugía de Bogotá firmó un convenio con la Universidad Colegio Mayor de Nuestra Señora del Rosario, para reabrir la antigua facultad de medicina que había cerrado sus puertas a finales del siglo XIX. Esta unión terminó a mediados de la última década del siglo pasado y es entonces cuando la Sociedad de Cirugía de Bogotá abre su propia facultad de medicina, dependiente de su brazo académico, la Fundación Universitaria de Ciencias de la Salud.

A los dos años de esta fundación reaparece Repertorio de Medicina y Cirugía con el número uno del volumen nueve en agosto de 2000 . Hoy, seis años después de demostrar la validez de este proyecto editorial, Colciencias nos incluye en el Índice Bibliográfico Nacional. Con ello, reafirmamos nuestra permanente invitación a los investigadores en ciencias de la salud, para que plasmen en nuestras páginas su esfuerzo y dedicación.

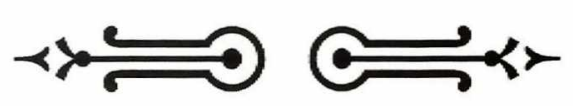

